\title{
Thermoelectric power of graphite intercalation compounds with iodine monobromide
}

\author{
Koji Kobayashi $^{\text {a),*}}$ and Ko Sugihara ${ }^{a),}$
}

\begin{abstract}
Graphite intercalation compounds (GICs) with interhalogens, iodine monochloride (ICl-GICs) and iodine monobromide (IBrGICs), are acceptor-type materials. They have a layer structure, and consequently, their thermoelectric power (TEP) along the basal plane (in-plane) is quite different from that in the vertical direction ( $c$-axis). The in-plane TEP shows an approximately linear temperature dependence at low temperatures, which is ascribed to the diffusion term, and levels off above $200 \mathrm{~K}$. This behavior stems from a phonon drag effect. However, the $c$-axis TEP shows approximately linear temperature dependence, which is a characteristic feature of the diffusion term. By choosing an appropriate set of parameters, the theory developed by Sugihara is found to give good agreement with the observed TEP results. We discuss the similarities and differences of both in-plane and $c$-axis TEP of IBr-GICs and ICl-GICs.
\end{abstract}

KEYWORDS : Graphite, Intercalation, Thermoelectric power, In-plane, c-axis

\section{Introduction}

Graphite intercalation compounds (GICs) have the staging structure and inserted intercalate layers periodically arrayed between graphite layers without destroying the stacking structure of graphite. Consequently, physical properties of GICs along the basal plane (inplane) behave quite differently from those along the vertical direction (c-axis).

GICs with interhalogens, iodine monochloride (ICl), and iodine monobromide (IBr), are typical examples of the acceptor GICs. These physical properties have been extensively studied ${ }^{1)-8}$. These studies proved that GICs have metallic properties, in contrast with the semimetallic one of host graphite.

In this work, in-plane TEP of the stage-2 IBr-GICs is measured between 5 to $300 \mathrm{~K}$, and the main purpose of the present work is to examine the similarities and differences of in-plane TEP behavior of different intercalants by comparing this result with in-plane TEP of ICl-GICs ${ }^{2}$. Observed TEP of this sample shows a linear increase with $T$ at low temperature, which is characteristic of the diffusion term. This behavior is similar to the TEP of ICl-GICs, carried out by us ${ }^{2}$. Above $100 \mathrm{~K}$, the TEP exhibits a saturation behavior which is attributed to the phonon drag effect. This saturation temperature is different from those of ICl-GICs. Three kinds of parameters $P_{i}, f$ and $B$ are included in the theory developed by Sugihara, ${ }^{2), 9)}$ where $P_{i}$ denotes an exponent of the relaxation time assumed in the form $\tau_{i}(E) \propto E_{i}^{P i}, f$ and $B$ are the parameters related to the phonon relaxation caused by strain field and/or point defects and vacancies, and by phonon-phonon interaction, respectively. These parameters of the stage-2 IBr-GICs are determined in comparison with the observed results.

$C$-axis TEP of the interhalogen $\mathrm{GICs}^{1), 3)-6)}$ shows approximately linear temperature dependence, which is a characteristic feature of the diffusion term. This is because in the acceptor GICs, the carrier conduction in $c$-axis direction is due to hopping conduction where carriers jump through conduction paths generated between the graphite layers ${ }^{9)-12)}$. Fig. 1 illustrates the mechanism for TEP of GICs.

\section{Specimen and Experimental Results}

Specimens used in the present study were prepared by synthesis of highly oriented pyrolytic graphite (HOPG) and IBr through the vapor transport method at $105 \mathrm{~K}$. By means of X-ray diffraction measurement and the mass measurement, the repeat distance along the $c$ direction as $I_{\mathrm{c}}=1.055 \mathrm{~nm}$, the chemical composition and density were obtained as $\mathrm{C}_{17.8} \mathrm{IBr}$ and $d=2.85 \times 10^{3} \mathrm{~kg} \mathrm{~m}^{-3}$, respectively.

Fig. 2 illustrates in-plane $(O)$ and $c$-axis $(\bigcirc)$ TEP of the stage-2 IBr-GICs $v s$. temperature $(T)$ curves. TEP $(S)$ is composed of the two contributions.

$S=S_{\mathrm{d}}+S_{\mathrm{p}}$ 


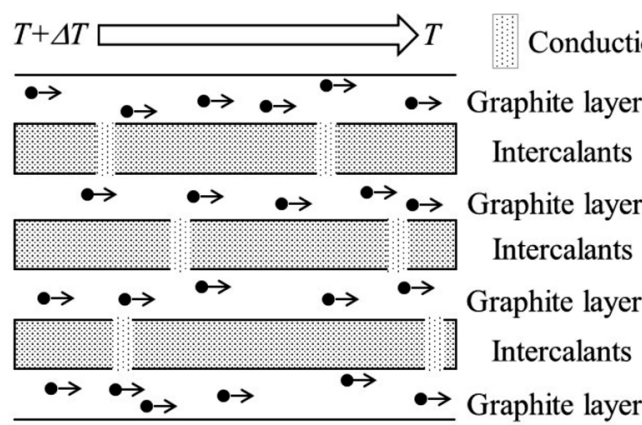

in-plane

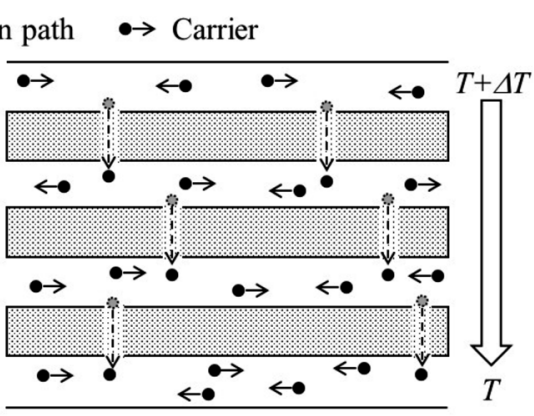

$c$-axis

Fig. 1 The mechanism for TEP of GICs.

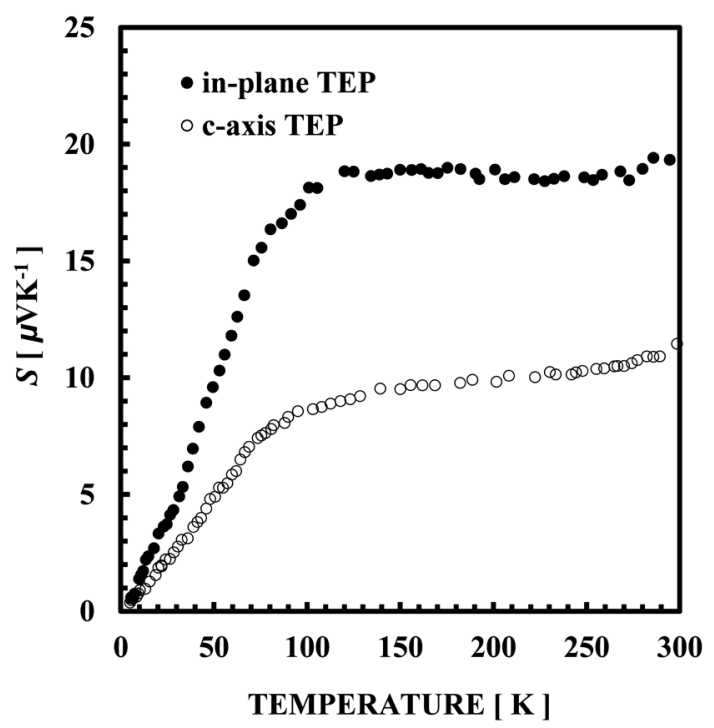

Fig. 2 The temperature behavior of in-plane $(0)$ and $c$-axis ${ }^{5), 6)}(\bigcirc)$ thermoelectric power of the stage-2 IBr-GICs.

where $S_{\mathrm{d}}$ is the diffusion term, and $S_{\mathrm{p}}$ denotes the phonon drag term. In-plane TEP curves in Fig. 2 shows a gradual slope change around $80 \sim 110 \mathrm{~K}$, though below and above this temperature range, it shows an almost linear temperature dependence. The result shows that at low temperatures $(\sim 30 \mathrm{~K})$ the contribution to $S$ mainly comes from $S_{\mathrm{d}}$ which shows approximate linear temperature dependence, while at intermediate temperatures $(30 \sim 120 \mathrm{~K}) S_{\mathrm{p}}$ indicating the phonon drag effect appears in addition to $S_{\mathrm{d}}$, and at high temperatures $(120 \mathrm{~K} \sim) S$ becomes saturated and in this region $S_{\mathrm{p}}$ turns into a decrease. However, $c$-axis TEP is $1.1 \times 10^{-5} \mathrm{~V} / \mathrm{K}$ at $300 \mathrm{~K}$, this is about half the value of in-plane TEP. Its curves shows an approximate linear temperature dependence, which is a characteristic feature of the diffusion term, though its slope changes gradually around $80 \sim 100 \mathrm{~K}^{5), 6)}$.

\section{In-plane Thermoelectric Power of IBr-GICs}

\subsection{Diffusion term of in-plane TEP}

In multi-carrier system $S$ is given by

$$
S=\frac{\sum_{i} \sigma_{i} S_{i}}{\sum_{i} \sigma_{i}}, \quad \sigma_{i}=\frac{N_{i} e^{2} \tau_{i} v_{\mathrm{F}_{i}}}{\hbar k_{\mathrm{F}_{i}}},
$$

where for the $i$-th carrier system, $\sigma_{i}$ is the partial electrical conductivity, $S_{i}$ the partial TEP, $\tau_{i}$ the partial relaxation time, $k_{\mathrm{F} i}$ represents the partial Fermi wave vector, $N_{i}$ is the carrier density,

$$
N_{i}=\frac{2 \times 2}{(2 \pi)^{3}} \frac{2 \pi}{I_{\mathrm{c}}} \pi k_{F_{i}}^{2}=\frac{k_{F_{i}}^{2}}{\pi I_{\mathrm{c}}},
$$

and $v_{\mathrm{F} i}$ the Fermi velocity,

$$
v_{\mathrm{F}_{i}}=\frac{1}{\hbar}\left(\frac{\partial E_{i}}{\partial k}\right)_{E_{i}}, \quad\left(k^{2}=k_{x}^{2}+k_{y}^{2}\right) .
$$

for the $i$-th carrier system, the diffusion TEP $S_{\mathrm{d} i}$ is given by

$$
S_{\mathrm{d}_{i}}=\frac{\pi^{2} k_{\mathrm{B}}^{2}}{3 e} T\left[\frac{\partial \log \sigma_{i}}{\partial E_{i}}\right]_{E_{i}},
$$

Using the Blinowski-Rigaux band model $^{13)}$ for the stage-2 compound, the dispersion relaxations $E_{1}$ and $E_{2}$ are obtained by changing the sign of the each hole band, ${ }^{13), 14)}$

$$
\begin{aligned}
& E_{1}=\frac{1}{2}\left[\left(\gamma_{1}^{2}+9 \gamma_{0}^{2} b^{2} k^{2}\right)^{1 / 2}-\gamma_{1}\right], \\
& E_{2}=\frac{1}{2}\left[\left(\gamma_{1}^{2}+9 \gamma_{0}^{2} b^{2} k^{2}\right)^{1 / 2}+\gamma_{1}\right],
\end{aligned}
$$

where $\gamma_{0}$ is the in-plane energy overlap parameter for neighboring carbon atoms, $\gamma_{1}$ is the out-of-plane energy overlap parameter for neighboring carbon atoms and $b$ is the $\mathrm{C}-\mathrm{C}$ distance $(0.142 \mathrm{~nm})$. By assuming the relaxation time $\tau_{i}^{2), 14)}$ as

$$
\tau_{1}=\tau_{01} E_{1}^{P_{1}}, \quad \tau_{2}=\tau_{02}\left(E_{2}-\gamma_{1}\right)^{P_{2}},
$$

eqs. (2) (4) and (6) lead to

$$
\begin{aligned}
& S_{\mathrm{d}_{1}}=\frac{\pi^{2} k_{\mathrm{B}}^{2}}{3 e} T\left[\frac{2 E_{\mathrm{F}}+\gamma_{1}}{E_{\mathrm{F}}^{2}+E_{\mathrm{F}} \gamma_{1}}+\frac{P_{1}}{E_{\mathrm{F}}}-\frac{2}{2 E_{\mathrm{F}}+\gamma_{1}}\right], \\
& S_{\mathrm{d}_{2}}=\frac{\pi^{2} k_{\mathrm{B}}^{2}}{3 e} T\left[\frac{2 E_{\mathrm{F}}-\gamma_{1}}{E_{\mathrm{F}}^{2}-E_{\mathrm{F}} \gamma_{1}}+\frac{P_{2}}{E_{\mathrm{F}}-\gamma_{1}}-\frac{2}{2 E_{\mathrm{F}}-\gamma_{1}}\right] .
\end{aligned}
$$

where $E_{\mathrm{F}}$ is the Fermi energy measured from $E_{1}=0$.

\subsection{Phonon drag term of in-plane TEP}

For the $i$-th carrier system, the phonon drag TEP $\left(S_{\mathrm{p}}\right)$ is in general represented by the following formula: ${ }^{15)}$ 


$$
S_{\mathrm{p}_{i}}=\frac{\left\langle C_{\mathrm{p}_{i}} R\right\rangle_{i}}{2 e N_{i}},
$$

where $C_{\mathrm{p} i}$ denotes the specific heat of the phonon system, which can interact with $\pi$-electron of GICs. $R$ is the momentum transfer ratio through the electron-phonon interaction from the phonon system to carriers, which is defined by

$$
R=\frac{t}{t_{\mathrm{c}}},
$$

where $t$ is the total phonon relaxation time included $t_{\mathrm{c}}$ which is the carrier-phonon relaxation time.

The phonon relaxation process plays an important role in the phonon drag effect. According to the theory developed by Sugihara, the total phonon relaxation rate $1 / t$ is presented by ${ }^{9)}$

$$
\frac{1}{t}=\frac{1}{t_{\mathrm{b}}}+\frac{1}{t_{\mathrm{c}}}+\frac{1}{t_{\mathrm{I}}}+\frac{1}{t_{\mathrm{p}}},
$$

where each component of relaxation rate is due to the following scattering processes.

(i) The relaxation rate $\left(1 / t_{\mathrm{b}}\right)$ due to the scattering caused at crystallite boundary,

$$
\frac{1}{t_{\mathrm{b}}} \simeq \frac{v_{\mathrm{s}}}{L} \equiv b,
$$

where $L$ is the crystallite dimension.

(ii) The relaxation rate $\left(1 / t_{c}\right)$ due to the scattering caused by the interaction between carrier and in-plane phonon. Ignoring the intraband transition, $1 / t_{\mathrm{c}}$ is given by

$$
\frac{1}{t_{\mathrm{c}}}=\sum_{i} \frac{1}{t_{i i}}=\sum_{i} \frac{\alpha_{i i} \hbar q k_{F i}}{\pi^{2} I_{\mathrm{c}} p_{0}^{2}} \equiv a q,
$$

where $p_{0}=(3 / 2) \gamma_{0} b, \alpha_{i i}=\pi D^{2} / d, D$ is the coupling constant between carrier and in-plane phonons and $d$ is the density.

(iii) The relaxation rate $\left(1 / t_{\mathrm{I}}\right)$ due to Rayleigh scattering ${ }^{16)}$ associated with point defects and vacancies and also due to the strain-field scattering, where both scattering processes give rise to the same $q$ dependence. For two-dimensional phonons, $1 / t_{\mathrm{I}}$ is given by

$$
\frac{1}{t_{\mathrm{I}}}=f q^{3} \text {. }
$$

(iv) The relaxation rate $\left(1 / t_{\mathrm{p}}\right)$ due to the phonon-phonon scattering process, which for two-dimensional phonons has the functional form $^{15), 17), 18)}$ :

$$
\frac{1}{t_{\mathrm{p}}}=B q T^{3}
$$

From eqs. (12) (16), eq (11) becomes

$$
R=\frac{t}{t_{\mathrm{c}}}=\frac{a q}{b+a q+f q^{3}+B q T^{3}} .
$$

In eq. (10), $\left\langle C_{p i} R\right\rangle_{i}$ takes the form ${ }^{2), 9)}$ :

$$
\left\langle C_{p_{i}} R\right\rangle_{i}=\frac{1}{2 \pi I_{\mathrm{c}}} \int_{0}^{k_{F_{i}}} \mathrm{~d} q q \frac{\left(\hbar \omega_{q}\right)^{2}}{k_{\mathrm{B}} T^{2}} N_{q}\left(N_{q}+1\right) R(q),
$$

where $N_{q}$ is the equilibrium in-plane phonon distribution. Rewriting eq. (17) introducing $\hbar v_{\mathrm{s}} q_{\mathrm{T}}=k_{\mathrm{B}} T$ and $x=\hbar v_{\mathrm{s}} q / k_{\mathrm{B}} T$, we have

$$
\begin{aligned}
\left\langle C_{p_{i}} R\right\rangle_{i} & =\frac{k_{\mathrm{B}} q_{\mathrm{T}}^{2}}{2 \pi I_{\mathrm{c}}} \int_{0}^{x_{\max }} \mathrm{d} x x^{3} \frac{\mathrm{e}^{x}}{\left(\mathrm{e}^{x}-1\right)^{2}} R(x), \\
x_{\max _{i}} & =\frac{2 \hbar v_{\mathrm{s}} k_{\mathrm{F}_{i}}}{k_{\mathrm{B}} T} .
\end{aligned}
$$

From eqs. (3), (4) and (18) the phonon drag TEP for the stage-2 IBrGICs becomes

$$
\begin{aligned}
S_{\mathrm{p}_{i}} & =\frac{k_{\mathrm{B}}^{3}}{4 e k_{\mathrm{F}_{i}}^{2} \hbar^{2} v_{\mathrm{s}}^{2}} T^{2} \int_{0}^{x_{\max }} \mathrm{d} x x^{3} \frac{\mathrm{e}^{x}}{\left(\mathrm{e}^{x}-1\right)^{2}} R(x), \\
x_{\text {max }_{i}} & =\frac{2 \hbar v_{\mathrm{s}} k_{\mathrm{F}_{i}}}{k_{\mathrm{B}} T}(i=1,2) .
\end{aligned}
$$

\subsection{Comparison between calculated results and observed} in-plane TEP

In stage-2 GICs eq. (2) becomes

$$
S=\frac{\sigma_{1} S_{1}+\sigma_{2} S_{2}}{\sigma_{1}+\sigma_{2}} .
$$

It is needed to estimate the ratio $\sigma_{1} / \sigma_{2}$. Equations (2), (3) and (4) lead to

$$
\frac{\sigma_{1}}{\sigma_{2}}=\frac{k_{\mathrm{F}_{1}}^{2} k_{\mathrm{F}_{1}}^{P_{1}} k_{\mathrm{F}_{1}}\left(2 E_{\mathrm{F}}-\gamma_{1}\right) k_{\mathrm{F}_{2}}}{k_{\mathrm{F}_{2}}^{2} k_{\mathrm{F}_{2}}^{P_{2}} k_{\mathrm{F}_{2}}\left(2 E_{\mathrm{F}}-\gamma_{1}\right) k_{\mathrm{F}_{1}}}=\frac{k_{\mathrm{F}_{1}}^{P_{1}+2}\left(2 E_{\mathrm{F}}-\gamma_{1}\right)}{k_{\mathrm{F}_{2}}^{P_{2}+2}\left(2 E_{\mathrm{F}}-\gamma_{1}\right)} .
$$

At low temperatures we take the same assumption $P_{1}=P_{2}=0$ employed by Issi et al. ${ }^{2), 14)}$ At high temperature range, we have no information on the ratio $\sigma_{1} / \sigma_{2}$, where $P_{1} \neq 0$ and $P_{2} \neq 0$ are expected since the phonon scattering participates in the scattering process. Thus, we have

$$
\frac{\sigma_{1}}{\sigma_{2}}=\frac{k_{\mathrm{F}}^{P_{1}+2}\left(2 E_{\mathrm{F}}-\gamma_{1}\right)}{k_{\mathrm{F}_{2}}^{P_{2}+2}\left(2 E_{\mathrm{F}}-\gamma_{1}\right)}=1.42 .
$$

For simplicity, we use the assumption that eq. (22) holds at high temperatures. Combining eq. (22) with (21) we have

$$
\frac{k_{\mathrm{F}_{1}}^{P_{1}}}{k_{\mathrm{F}_{2}}^{P_{2}}}=1 \text { and } \frac{P_{1}}{P_{2}}=0.97 \text {. }
$$

In calculating TEP, the following parameters are taken from the graphite:

$$
\begin{aligned}
& \gamma_{0}=3.16 \mathrm{eV}, \quad \gamma_{1}=0.38 \mathrm{eV}, \quad D=16 \mathrm{eV}, \\
& v_{\mathrm{s}}=2.1 \times 10^{4} \mathrm{~ms}^{-1}, \quad L \simeq 10^{-6} \mathrm{~m} .
\end{aligned}
$$

From the galvanomagnetic experiment by our unpublished work and Barati et al. ${ }^{8)}$, following parameters are obtained:

$$
\begin{aligned}
& E_{\mathrm{F}}=0.55 \mathrm{eV}, \quad k_{\mathrm{F}_{1}}=1.4 \times 10^{9} \mathrm{~m}^{-1}, \\
& k_{\mathrm{F}_{2}}=0.82 \times 10^{9} \mathrm{~m}^{-1} .
\end{aligned}
$$

Inserting these values into eqs. (12) and (13), we obtain following parameters for the relaxation rate $\left(1 / t_{\mathrm{b}}\right)$ and $\left(1 / t_{\mathrm{c}}\right)$ :

$$
b=2.1 \times 10^{10} \mathrm{~s}^{-1}, \quad a=1.4 \times 10^{1} \mathrm{~m}^{3} \mathrm{~s}^{-1} .
$$

Using these values for eqs. (8), (16) and (19), and adjusting values $P_{i}$, $f$ and $B$, we obtain a best set of these four parameters. 


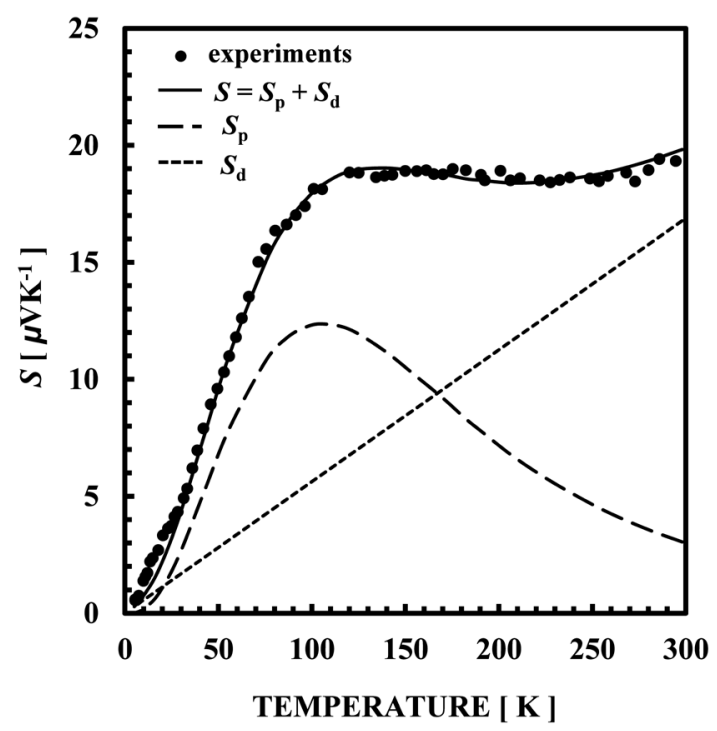

Fig. 3 Comparison of the theoretical calculation (solid line) and observed TEP ( ) for the stage-2 IBr-GICs. The dashed line $\left(S_{\mathrm{p}}\right)$ and the broken line $\left(S_{\mathrm{d}}\right)$ represent the calculated values for the phonon drag and the diffusion TEP.

$$
\begin{aligned}
& P_{1}=-0.18, \quad P_{2}=-0.19, \\
& f=2.4 \times 10^{-18} \mathrm{~m}^{3} \mathrm{~s}^{-1}, \quad B=6.0 \times 10^{-6} \mathrm{~m} \mathrm{~s}^{-1} \mathrm{~K}^{-3} .
\end{aligned}
$$

Fig. 3 shows the comparison between the observed TEP and the calculated results for the stage-2 IBr-GICs, where the black circles (O) correspond to the observed values, $S_{\mathrm{d}}$ and $S_{\mathrm{p}}$ represent the calculated values for the diffusion and phonon drag contribution, respectively. And the total calculated TEP is $S=S_{\mathrm{d}}+S_{\mathrm{p}}$.

Assuming $P_{1}=P_{2}=0$ and substituting eqs. (24), (25) into eq. (8) and applying eqs. (20), (22), the total diffusion TEP $S_{\mathrm{d}}$ is $2.1 \times 10^{-5} \mathrm{VK}^{-1}$ at $300 \mathrm{~K}$, which exceeds experimental results at room temperature range (Figs. 2, 3). However, by employing the values of $P_{1}$ and $P_{2}$ in (27) calculated TEP agrees well with experiments (Fig. 3). The total phonon relaxation rate obtained from these values of parameters $f$ and $B$ is equivalent to those obtained by Issi et al. ${ }^{19)}$ from the thermal conductivity of a similar acceptor type compound, the stage- $2 \mathrm{FeCl}_{3}$ GICs. Therefore, the values of (27) are considered appropriate.

\section{C-axis TEP of IBr-GICs}

$C$-axis thermoelectric power $\left(S_{\mathrm{c}}\right)$ is given by

$$
S_{\mathrm{c}}=\frac{\chi_{\mathrm{c}}}{T \sigma_{\mathrm{c}}}
$$

where $\sigma_{\mathrm{c}}$ is the $c$-axis conductivity ${ }^{4)-6), 20)}$, which results from hopping conduction along the conduction path present in $c$-axis direction $^{9)-12), 20)} \cdot \chi_{\mathrm{c}}$ is related to Peltier coefficient $\Pi_{\mathrm{c}}$ by $\chi_{\mathrm{c}}=\sigma_{\mathrm{c}} \Pi_{\mathrm{c}} \cdot \chi_{\mathrm{c}}$ is obtained from the expression for $\sigma_{\mathrm{c}}$ by replacing $e^{2} \rightarrow e\left(E_{k}-\zeta\right)^{20)}$. Using Eq. (6), the diffusion term of $c$-axis TEP $S_{\mathrm{c}-\mathrm{d}}$ is following expression,

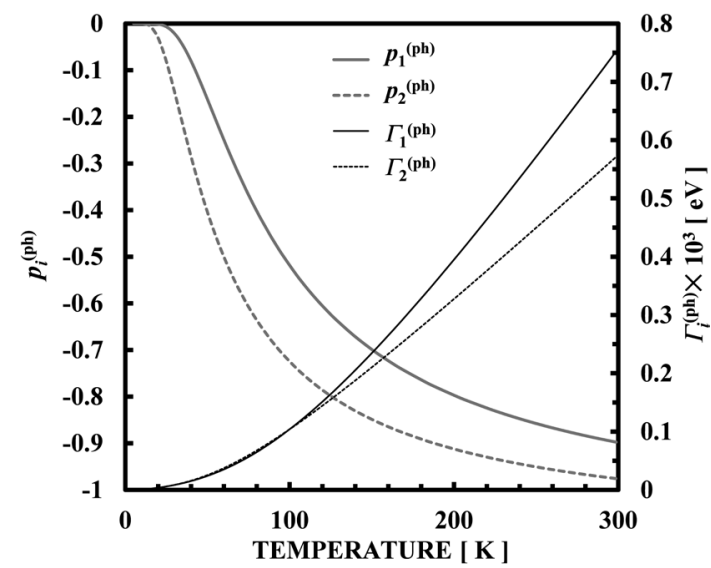

Fig. 4 Temperature dependence of $\Gamma_{i}^{\left({ }^{(p h)}\right.}$ and $p_{i}{ }^{(\mathrm{ph})}$ for the stage-2 IBr-GICs $^{6}{ }^{6}$. Reprinted with permission from ref. 6. Copyright 2000 Taylar \& Francis and Copyright Clearance Center.

$$
\begin{aligned}
& S_{\mathrm{c}-\mathrm{d}}= \pm \frac{\pi}{3 e} k_{\mathrm{B}}^{2} T \frac{\sum_{i=1}^{2} \sum_{m} \Gamma_{i}^{(m)}\left(1+p_{i}^{(m)}\right) / \Gamma_{i}^{2}}{\sum_{i=1}^{2}\left(E_{\mathrm{F}}+\Delta_{i}\right) / \Gamma_{i}}, \\
& \Delta_{i}=\left\{\begin{array}{c}
\gamma_{1} / 2: i=1 \\
-\gamma_{1} / 2: i=2
\end{array}, \quad \Gamma_{i}=\sum_{m} \Gamma_{i}^{(m)},\right. \\
& \Gamma_{i}^{(m)}=\frac{\hbar}{2 \tau_{i}^{(m)}} \propto E^{p_{i}^{(m)}},
\end{aligned}
$$

where $1 / \tau_{i}^{(m)}$ denotes the $m$-th scattering rate $i$-th band in basal plane, and $p_{i}^{(m)}$ takes the following values scattering to various scattering mechanisms ${ }^{20)}$. (i) $\Gamma_{i}^{(\mathrm{DH})}$ and $p_{i}^{(\mathrm{DH})}$ denote the Daumas-Hérold boundary scattering, $p_{i}^{(\mathrm{DH})}=0$ and $\Gamma_{1}^{(\mathrm{DH})} \approx \Gamma_{2}^{(\mathrm{DH})}$, (ii) $\Gamma_{i}^{(\mathrm{Sh})}$ and $p_{i}^{(\mathrm{Sh})}$ the short range potential scattering, $p_{i}^{(\mathrm{Sh})}=-1$, (iii) $\Gamma_{i}^{(\mathrm{Io})}$ and $p_{i}^{(\mathrm{Io})}$ the ionized impurity scattering, $p_{i}^{(\mathrm{Io})}=1+2 \Delta / E_{\mathrm{F}}$, (iv) $\Gamma_{i}^{(\mathrm{ph})}$ and $p_{i}^{(\mathrm{ph})}$ the inplane phonon scattering, $p_{i}^{(\mathrm{ph})}=-1$ at high temperature and $p_{i}^{(\mathrm{ph})}=0$ at low temperature. $\Gamma_{i}^{(m)}$ except $\Gamma_{i}^{(\mathrm{ph})}$ are temperature-independence, while $\Gamma_{i}{ }^{\left(\mathrm{ph}^{2}\right)}$ and $p_{i}{ }^{(\mathrm{ph})}$ is given by ${ }^{3)}$

$$
\begin{aligned}
& \Gamma_{i}^{(\mathrm{ph})}=\frac{\hbar D^{2}}{4 \pi v_{\mathrm{s}}} \frac{E_{i}+\Delta_{i}}{p_{0}^{2} k_{\mathrm{F}_{i}}} \int_{0}^{2 k_{\mathrm{F}_{\mathrm{F}}}} \mathrm{d} q \frac{q}{\sqrt{1-\left(q / 2 k_{\mathrm{F}_{i}}\right)^{2}}} \frac{1}{\mathrm{e}^{\beta \hbar \omega_{q}}-1}, \\
& p_{i}^{(\mathrm{ph})}=-\left[E_{i} \frac{\mathrm{d} \Gamma_{i}^{(\mathrm{ph}) / \mathrm{d} E_{i}}}{\Gamma_{i}^{(\mathrm{ph})}}\right]_{E_{i}=E_{\mathrm{F}}}, \quad \beta=\frac{1}{k_{\mathrm{B}} T} .
\end{aligned}
$$

Fig. 4 shows the calculated results for $\Gamma_{i}^{(\mathrm{ph})}$ and $p_{i}^{(\mathrm{ph})}$, where values of eqs. (24) and (25) are employed. With increasing temperature, the factor $1+p_{i}^{\left({ }^{(p h)}\right.}$ in eq. (29) becomes small. This is reason why the $S_{\mathrm{c}} v s$. $T$ curves exhibits the slope change as is shown in Fig. 2.

Fig. 5 shows the comparison between the observed $S_{\mathrm{c}}$ and the calculated results $S_{\mathrm{c}-\mathrm{d}}$ for the stage-2 $\mathrm{IBr}_{-}^{-5), 6)}$ and $\mathrm{ICl}_{-}{ }^{4)} \mathrm{GICs}$, where fitting parameters $\Gamma_{i}^{(\mathrm{DH})}, \Gamma_{i}^{(\mathrm{sh})}$ and $\Gamma_{i}^{(\mathrm{Io})}$ are chosen as follows, respectively (in eV unit): 


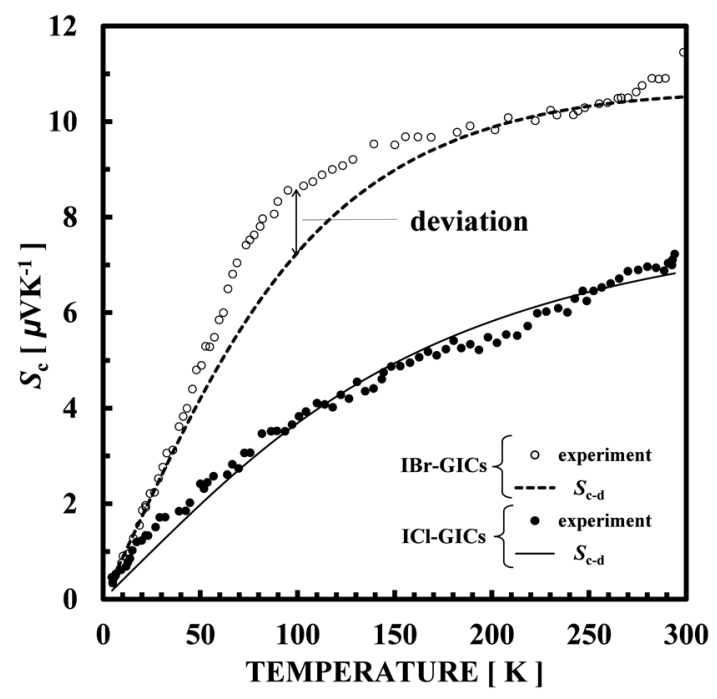

Fig. 5 Comparison of the observed $c$-axis TEP and the theoretical calculated $c$-axis diffusion TEP for the stage-2 IBr-GICs and ICl-GICs $^{6}$. The deviation shows the difference between the observed $c$-axis TEP and the calculated diffusion one for stage-2 IBr-GICs. Reprinted with permission from ref. 6 . Copyright 2000 Taylar \& Francis and Copyright Clearance Center.

$$
\left.\begin{array}{l}
\Gamma_{1}^{(\mathrm{DH})}=\Gamma_{2}^{(\mathrm{DH})} \cong 0, \quad \Gamma_{1}^{(\mathrm{Sh})}=\Gamma_{2}^{(\mathrm{Sh})} \cong 0, \\
\Gamma_{1}^{(\mathrm{Io})}=5.5 \times 10^{-4}, \quad \Gamma_{2}^{(\mathrm{Io})}=2.7 \times 10^{-4}, \quad \\
\Gamma_{1}^{(\mathrm{DH})}=\Gamma_{2}^{(\mathrm{DH})}=4.67 \times 10^{-4}, \\
\Gamma_{1}^{(\mathrm{Sh})}=3.26 \times 10^{-4}, \\
\Gamma_{2}^{(\mathrm{Sh})}=1.97 \times 10^{-4}, \quad \Gamma_{1}^{(\mathrm{Io})}=9.20 \times 10^{-4}, \quad(\text { for IBr-GICs }),{ }^{5), 6)} \\
\Gamma_{2}^{(\mathrm{Lo})}=5.56 \times 10^{-4}
\end{array}\right\}(\text { for ICl-GICs }) .{ }^{4)}
$$

In Fig. 5, though the curves $S_{\mathrm{c}-\mathrm{d}}$ for the stage-2 ICl-GICs reproduce the observed TEP, however, the curves $S_{\mathrm{c}-\mathrm{d}}$ for the stage-2 IBr-GICs can not reproduce the observed TEP about $50-180 \mathrm{~K}$. We assume this deviation between $S_{\mathrm{c}-\mathrm{d}}$ and $S_{\mathrm{c}}$, stems from the phonon drag effect. Therefore, we attempt to calculate the phonon drag TEP as follows.

Before proceeding to the discussion on TEP we consider about the phonon relaxation processes in $c$-direction, which play an important in the phonon drag effect. The total phonon relaxation rate is represented by eq. (11) similarly to the in-plane TEP. Though the phonon drift velocity along the $c$-direction is zero in the absence of a temperature gradient, however, it becomes finite through the interaction with carriers drifting along the $c$-axis, therefore it is given by

$$
v_{\mathrm{c}-\mathrm{ph}}=R(q) v_{\mathrm{c}-\mathrm{d}}, \quad v_{\mathrm{c}-\mathrm{d}}=\mu_{\mathrm{c}} F,
$$

where $v_{\mathrm{c}-\mathrm{d}}$ is the drift velocity of carrier and $\mu_{\mathrm{c}}$ denotes the $c$-axis mobility and $F$ the corresponding electric field. In contrast with $R(q)$ for the in-plane phonons mentioned the previous section, we have no theory on $R(q)$, where the out-of-plane phonons play a dominant role in the present case. Then, we introduce the following assumption. Since the interaction between carriers and the out-of-plane phonons is weak, the numerator $a q\left(1 / t_{\mathrm{c}}\right)$ is taken as a constant. The remaining

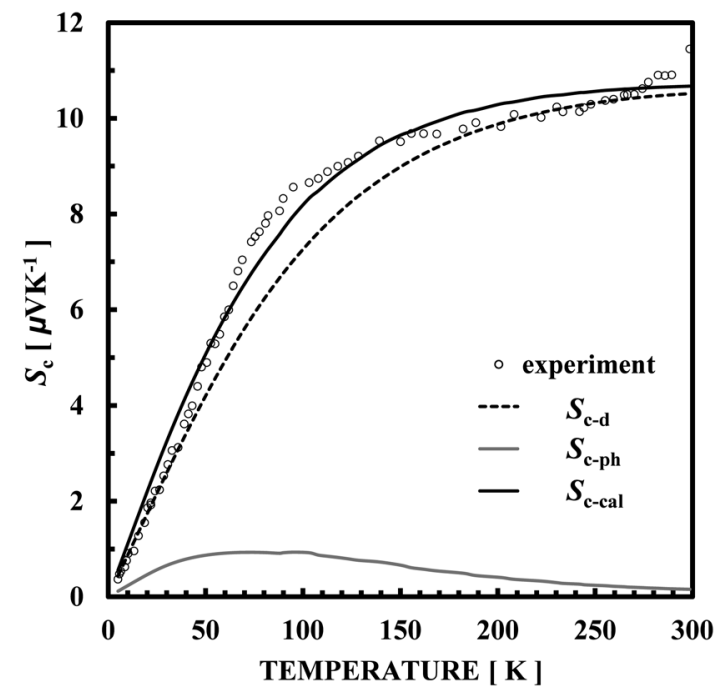

Fig. 6 Comparison of the theoretical calculation (solid line) and the observed $c$-axis TEP $(\bigcirc)$ for the stage-2 IBr-GICs. The dashed line $\left(S_{\mathrm{c}-\mathrm{d}}\right)$ and the shadow line $\left(S_{\mathrm{c}-\mathrm{ph}}\right)$ represent the calculated values for the diffusion and the phonon drag $\mathrm{TEP}^{5}$. Reprinted with permission from ref. 5. Copyright 1999 The Physical Society of Japan.

two terms corresponding to $1 / t_{\mathrm{I}}$ and $1 / t_{\mathrm{p}}$ processes are averaged over $T$ and replaced by a term proportional to $T^{P}(P>1)$. Therefore, in the case of $c$-axis conduction, we assume $R(q) \rightarrow R_{\mathrm{c}}(T)$, in the following form:

$$
R_{\mathrm{c}}(T)=\left(A+B T^{P}\right)^{-1}, \quad P>1,
$$

where $A, B$ and $P$ are fitting parameters. After long calculations, $c$ axis phonon drag TEP of GICs is given by

$S_{\mathrm{c}-\mathrm{ph}}=\frac{R_{\mathrm{c}}\left(k_{\mathrm{B}} T\right)^{3}}{4 \pi^{2} n e \hbar v_{\mathrm{c}} k_{\mathrm{B}} T^{2}} \frac{\sum_{i=1}^{2} \frac{E_{\mathrm{F}}+\Delta_{i}}{\Gamma_{i}}\left\langle q_{a i}^{2}\right\rangle}{\sum_{i=1}^{2} \frac{E_{\mathrm{F}}+\Delta_{i}}{\Gamma_{i}}} \int_{0}^{X_{m}} \mathrm{~d} X_{m} \frac{X^{2} \mathrm{e}^{X}}{\left(\mathrm{e}^{X}-1\right)^{2}}$,

$X_{m}=\frac{\hbar v_{\mathrm{c}} q_{m}}{k_{\mathrm{B}} T}, \quad q_{m}=\frac{2 \pi}{I_{\mathrm{c}}}$

where $q_{a 1} \cong k_{\mathrm{F} 1}, q_{a 2} \cong k_{\mathrm{F} 2}$. Inserting $v_{\mathrm{c}}=3.5 \times 10^{3} \mathrm{~m} \mathrm{~s}^{-1}$ and several values into eq. (34), and adjusting the values of $A, B$ and $P$, we obtained a best set of these parameters:

$$
A=22, \quad B=5.7 \times 10^{-6}, \quad P=3.0,
$$

where inserting these values into eq. (33), it satisfies $R_{\mathrm{c}}<1$.

Fig. 6 shows the comparison between the observed and the calculation TEP, where the white circles $(\bigcirc)$ correspond to the observed values, $S_{\mathrm{c}-\mathrm{d}}$ and $S_{\mathrm{c} \text {-ph }}$ represent the calculated values for the diffusion and the phonon drag TEP, respectively. And the total calculated $c$-axis TEP is $S_{\mathrm{c}-\mathrm{cal}}=S_{\mathrm{c}-\mathrm{d}}+S_{\mathrm{c}-\mathrm{ph}}$.

\section{Discussion}

Analytical results for TEP of stage-2 IBr-GICs shown in Fig. $\mathbf{3}$ are 


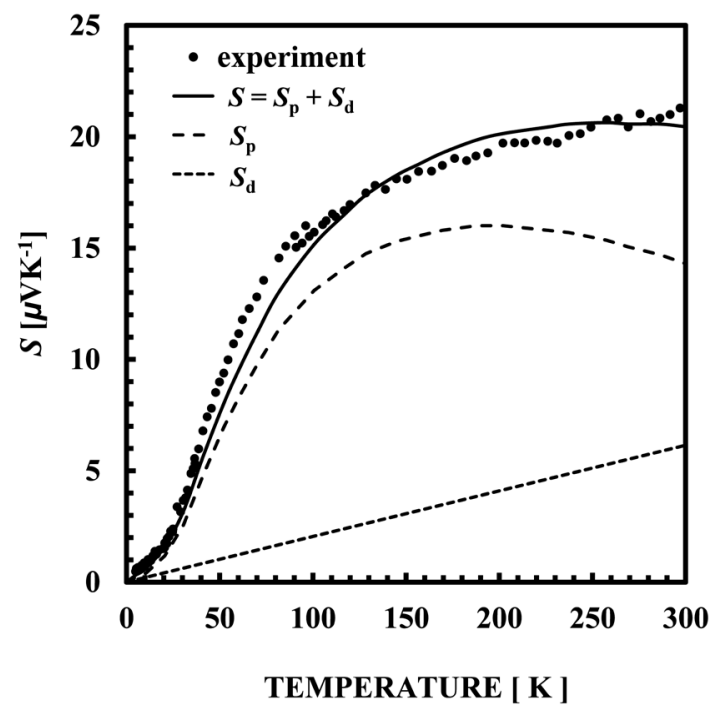

Fig. 7 Comparison of the theoretical calculation (solid line) and the observed TEP $(\mathcal{O})$ for the stage-2 ICl-GICs. The dashed line $\left(S_{\mathrm{p}}\right)$ and the broken line $\left(S_{\mathrm{d}}\right)$ represent the calculated values for the phonon drag and the diffusion $\mathrm{TEP}^{2}$.

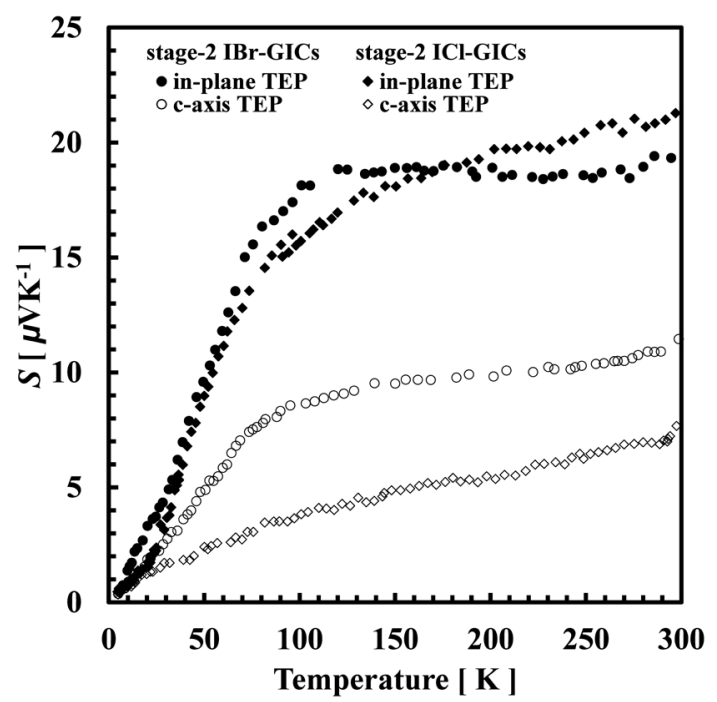

Fig. 8 The temperature behavior of in-plane $(O)$ and $c$-axis ${ }^{5), 6)}(\bigcirc)$ thermoelectric power of the stage-2 IBr-GICs, and in-plane ${ }^{2)}$ $(\diamond)$ and $c$-axis ${ }^{4)}(\diamond)$ thermoelectric power of the stage-2 ICl-GICs.

compared with those of stage-2 ICl-GICs shown in Fig. $7^{2}$. Carrier density of these specimens obtained from Hall effect measurements is almost identical to be about $8 \times 10^{26} \mathrm{~m}^{-3}{ }^{7,8)}$ but $S_{\mathrm{d}}$ of IBr-GICs is considerably larger than that of ICl-GICs. This difference is derived from the difference in $P_{i}$ values of Eq. (8) for these specimens and also considered to be due to the difference in $E_{\mathrm{F}}$ values: $0.55 \mathrm{eV}$ for IBr-GICs and $0.77 \mathrm{eV}$ for ICl-GICs.

Since boundary scattering $\left(1 / t_{\mathrm{b}}\right)$ is dominant below $50 \mathrm{~K}, S_{\mathrm{p}}$ is almost the same for these specimens at this temperature region. With increasing temperature, $S_{\mathrm{p}}$ of IBr-GICs increases and shows a clear peak at about $100 \mathrm{~K}$. On the other hand, $S_{\mathrm{p}}$ of ICl-GICs decreases gradually without showing a clear peak. This is because temperature sensitive Rayleigh scattering $\left(1 / t_{\mathrm{I}}\right)$ as well as phono-phonon scattering $\left(1 / t_{\mathrm{p}}\right)$ becomes more active in IBr-GICs than in ICl-GICs.

Fig. 8 shows experimental results of in-plane and $c$-axis TEP for both IBr-GICs and ICl-GICs. In-plane TEP is similar to each other for these specimens but $c$-axis TEP is significantly larger for $\mathrm{IBr}$ GICs than for ICl-GICs. $c$-axis TEP results from hopping conduction along the conduction path present in $c$-axis direction ${ }^{9)-12)}$ and the difference in $S_{\mathrm{d}}$ shown in Fig. 3 and Fig. 7 leads to this significant difference of $c$-axis TEP. The theory developed by Sugihara ${ }^{2), 9)}$ is found to explain well TEP of interhalogen GICs.

\section{Summary}

The theory developed by Sugihara ${ }^{2), 9)}$ explains well TEP of IBrGICs by adjusting four parameters available in the theory. Below $170 \mathrm{~K}, S_{\mathrm{p}}>S_{\mathrm{d}}$ for in-plane TEP, indicating that phonon drag carrier transport is more dominant than diffusion at this temperature region.

$S_{\mathrm{p}}$ increases in proportional to $T^{2}$ in accordance with eq. (19) at low temperatures because boundary scattering $\left(1 / t_{\mathrm{b}}\right)$ is dominant and $R$ in eq. (16) is temperature insensitive. With increasing temperature, other scattering processes become active and $S_{\mathrm{p}}$ incrases linearly with temperature. $S_{\mathrm{p}}$ shows a clear peak against temperature at about $100 \mathrm{~K}$ and decreases above $100 \mathrm{~K}$. Because of it is mainly increase of phonon-phonon scattering $\left(1 / t_{\mathrm{p}}\right)$ that leads to this decrease of $S_{\mathrm{p}}$ above $100 \mathrm{~K}$.

$C$-axis TEP of stage-2 IBr-GICs exhibits a deviation from $T$-linear dependence above $80 \mathrm{~K}$ and its slope decrease gradually with increasing $T$. The theory of $c$-axis TEP for GICs developed by Sugihara explains well the observed TEP of stage-2 IBr-GICs by adjusting parameters $^{5), 6)}$.

\section{Acknowledgements}

The authors are grateful to Dr. A. Moore of Union Carbide Corporation for kindly supplying HOPG specimens, and also to Professor Takuro Tsuzuku and Professor Kiyoshi Kawamura for their interest and stimulating discussion.

We pray for the soul of the late Professor Ko Sugihara, one of the authors.

\section{References}

1) T. Enoki, Graphite Intercalation Compounds and Applications (T. Enoki, M. Suzuki and M. Endo, eds.) (2003) p.190, OXFORD, New York.

2) K. Kobayashi, H. Oshima, K. Sugihara and T. Tsuzuku, J. Phys. Soc. Jpn. 61 (1992) 596-601.

3) K. Kobayashi, K. Sugihara, H. Oshima and T. Tsuzuku, J. Phys. Soc. Jpn. 63 (1994) 4451-4455.

4) K. Kobayashi, K. Sugihara and H. Oshima, J. Phys. Chem. Solids 57 (1996) 931-933.

5) K. Kobayashi, S. Suzuki, H. Oshima and K. Sugihara, J. Phys. Soc. Jpn. 


\section{8 (1999) 2006-2009.}

6) K. Kobayashi, S. Suzuki, H. Oshima and K. Sugihara, Mol. Cryst. Liq. Cryst. 340 (2000) 143-148.

7) Y. Ohta, K. Kawamura and T. Tsuzuku, J. Phys. Soc. Jpn. 57 (1988) 196204.

8) M. Barati, P. K. Ummat and W. R. Datars, J. Phys. Condens. Matter 11 (1999) 5569-5576.

9) K. Sugihara, Phys. Rev. B Condens. Matter 28 (1983) 2157-2165.

10) K. Sugihara, Phys. Rev. B Condens. Matter 29 (1984) 5872-5877.

11) S. Shimamura, Synth. Met. 12 (1985) 365-370.

12) K. Sugihara,TANSO. 1988 [No.134] 198-210 [in Japanese].

13) J. Blinowski, N. Hy Hau, C. Rigaux, J.P. Vieren, R. Le Tollec, G. Furdin,
A. Hérold and J. Melin, J. Phys. 41 (1980) 47-58.

14) L. Piraux, M. Kinany-Alaoui, J.-P. Issi, A. Perignon, P. Pernot and R. Vangelisti, Phys. Rev. B Condens. Matter 38 (1988) 4329-4332.

15) T. Tsuzuku and K. Sugihara, Chemistry and Physics of Carbon, Vol. 12 (P. L. Walker and P. A. Thrower, eds.) (1975) p. 109, Dekker, New York.

16) P. Carruthers, Rev. Mod. Phys. 33 (1961) 92-138.

17) K. Sugihara, J. Phys. Soc. Jpn. 29 (1970) 1465-1470.

18) K. Sugihara, T. Takezawa, T. Tsuzuku, Y. Hishiyama and A. Ono, J. Phys. Chem. Solids 33 (1972) 1475-1486.

19) J.-P. Issi, J. Heremans and M. S. Dresselhaus, Phys. Rev. B Condens. Matter 27 (1983) 1333-1347.

20) K. Sugihara, J. Phys. Soc. Jpn. 62 (1993) 624-633. 